\title{
Effect of barium-coated halloysite nanotube addition on the cytocompatibility, mechanical and contrast properties of poly(methyl methacrylate) cement
}

\author{
This article was published in the following Dove Press journal: \\ Nanotechnology, Science and Applications \\ 12 June 2017 \\ Number of times this article has been viewed
}

\author{
Uday Jammalamadaka' \\ Karthik Tappa' \\ Jeffery A Weisman' \\ James Connor Nicholson ${ }^{2}$ \\ David K Mills',3 \\ 'Center for Biomedical Engineering \\ and Rehabilitation Science, \\ ${ }^{2}$ Nanosystems Engineering, \\ ${ }^{3}$ The School of Biological Sciences, \\ Louisiana Tech University, Ruston, \\ LA, USA
}

\begin{abstract}
Halloysite nanotubes (HNTs) were investigated as a platform for tunable nanoparticle composition and enhanced opacity in poly(methyl methacrylate) (PMMA) bone cement. Halloysite has been widely used to increase the mechanical properties of various polymer matrices, in stark contrast to other fillers such as barium sulfate that provide opacity but also decrease mechanical strength. The present work describes a dry deposition method for successively fabricating barium sulfate nanoparticles onto the exterior surface of HNTs. A sintering process was used to coat the HNTs in barium sulfate. Barium sulfate-coated HNTs were then added to PMMA bone cement and the samples were tested for mechanical strength and tailored opacity correlated with the fabrication ratio and the amount of barium sulfate-coated HNTs added. The potential cytotoxic effect of barium-coated HNTs in PMMA cement was also tested on osteosarcoma cells. Barium-coated HNTs were found to be completely cytocompatible, and cell proliferation was not inhibited after exposure to the barium-coated HNTs embedded in PMMA cement. We demonstrate a simple method for the creation of barium-coated nanoparticles that imparted improved contrast and material properties to native PMMA. An easy and efficient method for coating clay nanotubes offers the potential for enhanced imaging by radiologists or orthopedic surgeons.
\end{abstract}

Keywords: barium, bone cement, halloysite, imaging, PMMA, sintering

\section{Introduction}

Bone cement is composed of poly(methyl methacrylate) (PMMA) and has been used by orthopedic surgeons for more than 6 decades. ${ }^{1-3}$ The first use of PMMA bone cement was in 1958 when British orthopedic surgeon Sir John Charnley implanted a hip endoprosthesis using PMMA. ${ }^{4}$ PMMA allows for the implantation and fixation of prostheses to the bone and consists of $\sim 90 \%$ polymer, and the remaining material consists of salts of barium or zirconium powders that make the resulting product radio-opaque. ${ }^{2,5}$ The chemical structure of PMMA cement is made up of a polymer of methyl methacrylate and consists of solid and liquid phases. The solid phase consists of prepolymerized PMMA, a reaction initiator (benzoyl peroxide) and radio-opacifiers. The liquid phase consists of methyl methacrylate monomer, a reaction accelerator $(\mathrm{N}, \mathrm{N}-$ dimethyl-p-toluidine) and a reaction retardant (hydroquinone). ${ }^{6}$ To prepare PMMA bone cement, the solid and liquid phases are mixed. The powder quickly dissolves in the monomer and undergoes a polymerization reaction by a free radical mechanism
Correspondence: David K Mills

The School of Biological Sciences,

Louisiana Tech University, Room 214,

Carson Taylor Hall, I Adams Boulevard,

Ruston, LA 7I 272, USA

Tel +l 3182675644

Emaildkmills@latech.edu 
to form putty-like cement. The polymerizing fluid bonds together the spheres and as the liquid monomer polymerizes, the bone cement hardens. ${ }^{2,4}$

PMMA has a high elastic modulus and as an inert biomaterial does not form a chemical bond or integrate with surrounding bone tissue at the implant site, resulting in a weak interface between the bone cement and the adjacent bone. ${ }^{6,7}$ Particle wear debris, ${ }^{8,9}$ secondary fractures ${ }^{10,11}$ and loosening or even displacement of the PMMA bone cement ${ }^{12}$ are unwanted complications. The addition of radio-opacifiers and antibiotics may also lead to decreased mechanical properties and fracture of the bone cement, which result in aseptic loosening and infections. ${ }^{13,14}$ PMMA has been the subject of continuous product development in parallel with improvements in surgical techniques. ${ }^{14,15}$ A major focus has been on the inclusion and testing of various additives or fillers designed to improve or remediate the mechanical, thermal and biological deficiencies in conventional PMMA. ${ }^{16,17}$ Researchers have studied combinations of PMMA bone cement and several micro- and nanoparticles, including barium sulfate, ${ }^{18}$ carbon nanotubes, ${ }^{19,20}$ chitosan, ${ }^{21}$ collagen, ${ }^{22}$ clays, ${ }^{23,24}$ hydroxyapatite, ${ }^{25,26}$ magnesium oxide, ${ }^{27}$ silica ${ }^{28,29}$ and silver nanoparticles ${ }^{30}$ (refer the study by Lewis ${ }^{17}$ for an extensive review of these, and other additives, and their effects on PMMA).

Halloysite nanotubes (HNTs) are naturally occurring clay minerals composed of silica and alumina. ${ }^{31-33}$ The outside of the tube has a net negative charge due to the silica, while the inside has a net positive charge attributed to the alumina. ${ }^{32,33}$ This charge difference is due to the bonding pattern of the aluminum and silicon atoms about each other. The typical inner diameter, outer diameter and length of these tubes are 1-30, 30-50 and 100-2000 nm, respectively. ${ }^{32,33}$ Since HNTs are hydrophilic and have opposite surface polarizations for the inner and outer lumen, they can be easily filled or coated. ${ }^{34}$ This capability and the natural availability of these nanoparticles have made them an ideal additive for many nanotechnology applications. They are particularly well suited for the sustained release of various drugs and bioactive agents. ${ }^{34-36}$ Halloysite also has thermal and mechanical properties that are highly desirable in polymer composites, primarily for the reinforcement of low-density polymers. ${ }^{37,38}$ By varying the amount of halloysite and selecting applicable coatings, the thermal and mechanical properties of given polymers can be specifically tailored to the desired application. Wei et $\mathrm{al}^{24}$ used halloysite as a nanocontainer for release of an antibiotic in PMMA cement. Gentamicin-doped HNTs admixed in PMMA bone cement showed significantly better elution of the antibiotic, with the increase in elution increasing with the increase in nanotube loading, and inhibited the growth of Staphylococcus aureus but not on specimens of their control (an approved bone cement brand). ${ }^{24}$

Metal acetate or metal acetylacetonates are unique complexes that are commonly used in chemistry. The metal acetylacetonates are coordination complexes made up of a metal ion and acetylacetonate anion. ${ }^{39}$ Iron and gold are more commonly known groups, but many other metals are also used, such as barium, and are readily manufactured by the chemical industry. These compounds degrade between $200^{\circ} \mathrm{C}$ and $300^{\circ} \mathrm{C}$. Due to their thermal degradation, they make ideal candidates for coatings via sintering. ${ }^{39,40}$ Recently, a dry sintering process was used to coat the surface of HNTs with metal acetylacetonates - compounds primarily used in the synthesis of nanoparticles, as metal catalysts and as nuclear magnetic resonance (NMR) shift reagents. ${ }^{41}$ We showed that this method is capable of thermally decaying the metal acetylacetonate, resulting in a free positively charged metal ion that readily bonded to the negatively charged HNT exterior, resulting in metallic barium, iron and nickel coatings on the HNT surface.

In this study, our sintering process was used for coating the HNTs with barium acetylacetonate (BA), and the coated HNTs were then added to PMMA bone cement. The composite material properties were assessed, and the potential effects on cell viability and proliferation were assessed. BA coatings improved contrast and strengthened the PMMA bone cement. Cellular results also support the concept that barium-coated HNTs are neither cytotoxic nor impact cell proliferation and may provide additional capabilities to established materials such as PMMA bone cement.

\section{Materials and methods Materials}

HNTs, gentamicin sulfate and the visualization reagents used in this study were purchased from Sigma-Aldrich (St. Louis, MO, USA). BA was purchased from Fischer Scientific Company (Hampton, NH, USA). Three different PMMA bone cements, Bosworth Original Truliner (Skokie, IL, USA) and Wright's Orthoset Radiopaque bone cement of low viscosity (ORTHOSET ${ }^{\circledR} 3$ ) and high viscosity (ORTHOSET ${ }^{\circledR}$ Premier) (Warsaw, IN, USA), were used.

Spectrophotometric analyses were performed on a Nanodrop spectrophotometer (Thermo Scientific, Wilmington, DE, USA). A Vulcan ${ }^{\circledR}$ A550 Series Bench Top Muffle Furnaces from Thomas Scientific (Swedesboro, NJ, USA) was used for heating the materials. 


\section{BA sintering}

BA was mixed with halloysite in a 1:1 ratio by weight. The mixing was done in 100-250 mg batches of halloysite to allow for a more uniform sintering. Halloysite was dried overnight at $60^{\circ} \mathrm{C}$. The halloysite was then weighed and mixed with an equal amount of a metal acetate or metal acetylacetonate. Powders were moderately mixed in a mortar and pestle. The powders were then placed into $5 \mathrm{~mL}$ borosilicate glass containers and vortexed vigorously for a minute. Containers were then placed in a Vulcan oven. The oven was programmed to heat to $300^{\circ} \mathrm{C}$ at a rate of $5^{\circ} \mathrm{C} / \mathrm{min}$. The program was then set to stay at this temperature for 4 hours, and the samples were then cooled to room temperature at $5^{\circ} \mathrm{C} / \mathrm{min}$. This resulted in a sintering reaction that coated HNTs in a metal shell or deposited metal clusters onto portions of the nanotube. After sintering, the glass tubes were vortexed, and the samples were ground by mortar and pestle to break up any clumps of material.

\section{Fabrication of BA-sintered nanoparticle enhanced bone cement}

HNTs were mixed into PMMA bone cement at 5\%, 7.5\%, $10 \%, 15 \%$ and $20 \% \mathrm{w} / \mathrm{w}$ concentration. PMMA bone cement, with and without HNTs, was then molded into different shapes for imaging and further characterization and mechanical testing (see subsequently for details). Bosworth Original Truliner is composed of all the regular components of PMMA cement except for radio-opacifiers. Orthoset Radiopaque bone cements are regular PMMA cements without antibiotic additives. All bone cement samples were prepared by mixing two parts of the powder content and one part of liquid monomer in a bowl.

\section{Material characterization and image analysis}

The surface microstructures of doped and undoped PMMA cement were characterized using a HITACHI S-4800
Field-Emission Scanning Electron Microscope (FE-SEM) (Chiyoda, Tokyo, Japan). Samples were attached to a conductive adhesive tape and placed onto a stage for viewing. A gold sputter coating $(4 \mathrm{~nm})$ was applied to the sample surfaces with a 208 HR Metal Sputter Coater (Cressington, Watford, England). The prepared stages were then placed into the sample chamber and viewed at $1.0-2.0 \mathrm{kV}$.

\section{Magnetic resonance imaging (MRI)}

For assessment of potential gains in imaging contrast, we tested nine PMMA cement formulations. Control groups consisted of commercial grade PMMA bone cement (Orthoset Radiopaque) and included low-viscosity (Group \#1) and high-viscosity cement samples (Group \#2). Additionally, plain PMMA powder was used to create bone cement constructs that lacked barium sulfate (Group \#3). Our experimental group samples were made using the PMMA-only bone cement. These were PMMA cement with $5 \%, 10 \%$ and $20 \% \mathrm{wt} /$ uncoated HNTs (Groups \#4, 5 and 6, respectively) and with $5 \%, 10 \%$ and $20 \% \mathrm{wt} /$ barium-coated HNTs (Groups \#7, 8 and

9 , respectively). All cement samples were mixed and placed within a dog-bone mold for setting. Control groups and experimental groups are shown in Table 1 . A $30 \mathrm{~mm}$ long rectangular bar sample of each cement sample was run through an MRI machine set to $120 \mathrm{kV}$. The three-dimensional (3D) scan was manually observed. Fifty to one hundred $1 \mathrm{~mm}$ point regions of interest (ROIs) were calculated in each sample for contrast and expressed in Hounsfield units.

\section{Compression testing}

For compression testing, PMMA cement, with and without HNTs, was molded into cylindrical pillars of $12 \mathrm{~mm}$ height and $6 \mathrm{~mm}$ radius using wax molds. These specimens $(\mathrm{N}=5$ / group) were produced per ASTM F451 standard for compression testing. Compression testing was performed using a Tinius Olsen universal testing machine in compression testing mode.

Table I Bone cement contrast levels as measured in Hounsfield units

\begin{tabular}{|c|c|c|c|c|c|}
\hline Control groups & $\begin{array}{l}\text { Hounsfield } \\
\text { units }\end{array}$ & HNTs & $\begin{array}{l}\text { Hounsfield } \\
\text { units }\end{array}$ & $\begin{array}{l}\text { Barium-coated } \\
\text { HNTs }\end{array}$ & $\begin{array}{l}\text { Hounsfield } \\
\text { units }\end{array}$ \\
\hline \multirow[t]{2}{*}{ Group \#I low viscosity } & 1473 & Group \#4 & 140 & Group \#7 & 382 \\
\hline & & $5 \% w t$ & & $5 \% w t$ & \\
\hline \multirow[t]{2}{*}{ Group \#2 high viscosity } & 1443 & Group \#5 & 164 & Group \#8 & 652 \\
\hline & & $10 \% w t$ & & $10 \% \mathrm{wt}$ & \\
\hline \multirow[t]{2}{*}{ Group \#3 PMMA only } & 68 & Group \#6 & 164 & Group \#9 & 1029 \\
\hline & & $20 \%$ wt & & $15 \% w t$ & \\
\hline
\end{tabular}

Note: $\mathrm{n}=3$ samples/per group tested.

Abbreviations: HNTs, Halloysite nanotubes; PMMA, poly(methyl methacrylate). 


\section{Flexural testing}

A three-point bending test was performed to assess the flexural strength of the fabricated PMMA samples ( $\mathrm{N}=5 /$ group), with and without HNTs. Bar-shaped samples were molded in $75 \mathrm{~mm} \times 10 \mathrm{~mm} \times 5 \mathrm{~mm}$. The distance between adjacent points was set to $20 \mathrm{~mm}$, and the load speed was $0.75 \mathrm{~mm} /$ min. Flexural strength was calculated using the formula $\sigma=$ $3 f l / b d^{2}$, where $f$ is the peak force, $l$ is the span between the supports, $b$ is the width and $d$ is the thickness of the specimen.

\section{Osteosarcoma cell culture}

PMMA cement, barium sulfate-doped PMMA, PMMA with $10 \% \mathrm{w} / \mathrm{w}$ HNTs and BA-coated HNTs were fabricated to form thin disks of $1 \mathrm{~mm}$ thickness and $5 \mathrm{~mm}$ diameter and placed within a 96-well dish. Osteosarcoma cells (CRL 2836; American Type Culture Collection, Manassas, VA, USA) were chosen as our study model cell type. In many similar studies, osteoblast cells are the standard cell type used in cell viability tests on bone cements. The potential of halloysite as a nanocontainer for the sustained release of drug delivery has long been established. ${ }^{19,22,28}$ We envision coupling coated and drug-doped HNTs (e.g., methotrexate) as a potential drug delivery system.

Osteosarcoma cells seeded onto the cement disk surfaces, and the cell cultures were maintained in Dulbecco's Modified Eagle Medium containing 10\% fetal bovine serum and $1 \%$ penicillin-streptomycin-amphotericin. Cells cultured on cell culture plastic and disks containing PMMA only serve as controls. Live/Dead cytotoxicity assay $(n=6)$ was done to visualize cell viability, and osteosarcoma cell proliferation was evaluated using an 2,3-bis-(2-methoxy-4-nitro5-sulfophenyl)-2H-tetrazolium-5-carboxanilide (XTT)-assay $(n=6)$. Both assays were performed at 24 hours and 4 days after cell seeding.

\section{Live/dead assay}

Dyes for visualizing alive and dead cells were used for this assay. According to the kit protocol, $2 \mu \mathrm{M}$ solutions of dyes were added to test wells and incubated for 30 minutes. To assess any potential cytotoxicity effects of the different cement, the Live/Dead assay (Life Technologies, Carlsbad, CA, USA) was performed on six groups with three groups serving as controls. The three control groups consisted of 1) nonbone cement scaffold (serving as the negative control), 2) bone cement with no fillers (PMMA only) and 3) lowviscosity radiopaque cement (as positive control). The three experimental groups were 1) bone cement scaffolds containing $10 \% \mathrm{w} / \mathrm{w}$ barium-coated HNTs, 2) barium sulfate pow- der and 3) HNTs. Images taken were restricted to the outer edge of the scaffolds because of their opaqueness and were captured using an Olympus BX51 fluorescence microscope.

\section{XTT assay}

Cell proliferation was estimated using XTT assay (SigmaAldrich, MO, USA). The XTT dye (2,3-bis[2-Methoxy-4-nitro-5-sulfophenyl]-2-tetrazolium-5-carboxyanilide sodium salt) is reduced to an orange color dye by the dehydrogenase enzyme present in the mitochondria of living cells. Based on this principle, the assay was performed by following the protocol given by the manufacturer. Cells were assayed after day 1 and day 4 in culture. Photometric readings were taken after 4 hours of incubation in the XTT dye.

\section{Statistical analysis}

All the experiments were done in triplicate. Statistical analysis was carried out via one-way analysis of variance (ANOVA) tests, using MINITAB software v 16. $p<0.05$ was considered statistically significant. Data are expressed as mean \pm standard deviation (SD).

\section{Results}

\section{Coating HNTs}

Metal acetylacetonates were used to create custom metal deposition on HNTs through a sintering process. A major change in color was achieved with PMMA

An SEM of uncoated HNTs (Figure 1A) and bariumcoated HNTs (Figure 1B) demonstrates that HNTs were coated with barium. Barium coatings typically covered most of the HNTs' outer surface (Figure 1B). Energy dispersive spectroscopy (EDX) was run to analyze the composition of the barium-coated HNTs compared with control samples (uncoated HNTs). A barium peak indicating the presence of barium on the HNT surface is shown in Figure 1C.

\section{MRI}

MRI was used to assess the amount of contrast achieved with PMMA doped with barium-coated HNTs compared with commercial grade PMMA bone cement (Figure 2). One millimeter point ROIs were calculated for each sample for contrast in Hounsfield units, and the ROIs from the samples were averaged with values expressed in Hounsfield units (Table 1).

The PMMA-only cement has very low contrast and visibility at $68 \mathrm{HU}$. According to the literature, HNTs can add strength to PMMA but provide little contrast when compared to commercial low- or high-viscosity cement. In 

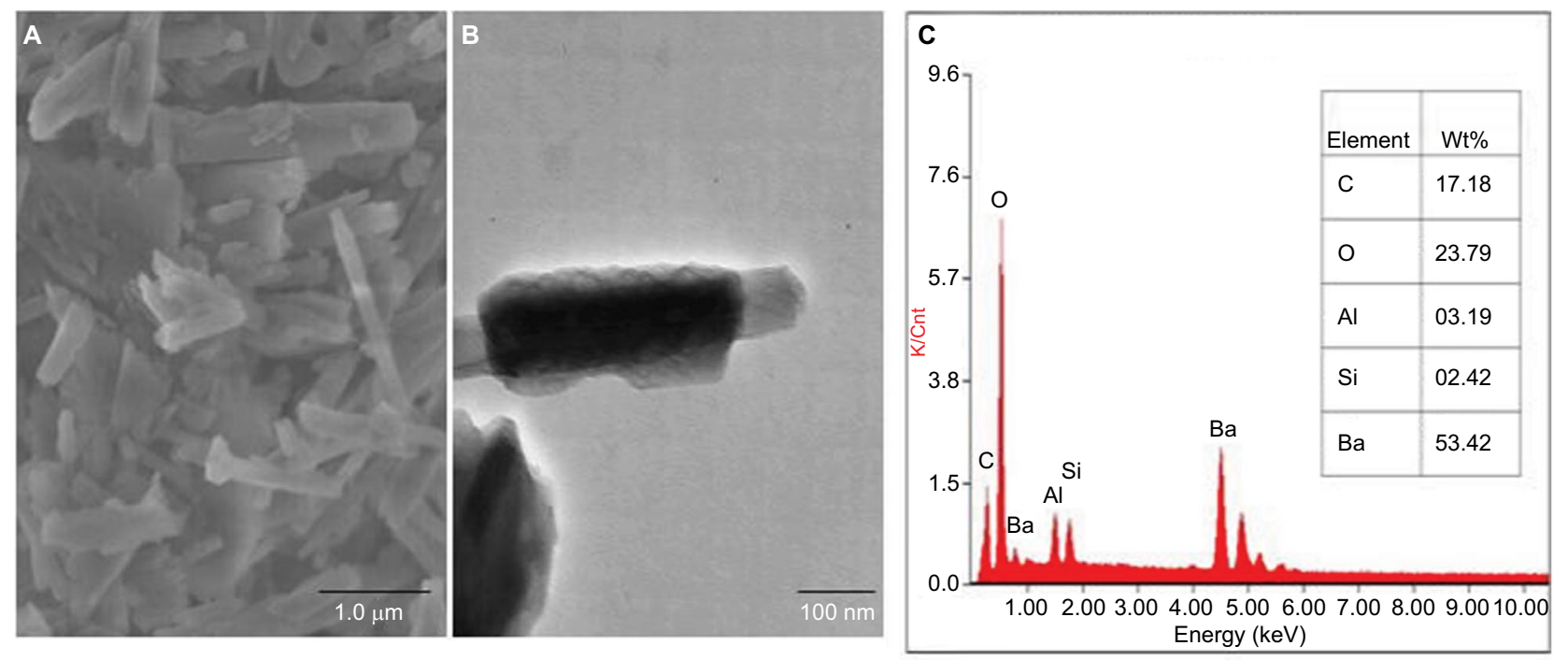

Figure I Analysis of barium-coated HNTs.

Notes: (A) SEM showing uncoated HNTs. (B) SEM showing a single barium-coated HNT, bars = I $\mu$ m and I00 nm, respectively. (C) EDX of barium-coated HNTs. The particles had to be secured with carbon tape to prevent damage to the SEM. (Carbon peaks are derived from the carbon tape.)

Abbreviations: HNTs, Halloysite nanotubes; SEM, scanning electron microscopy.

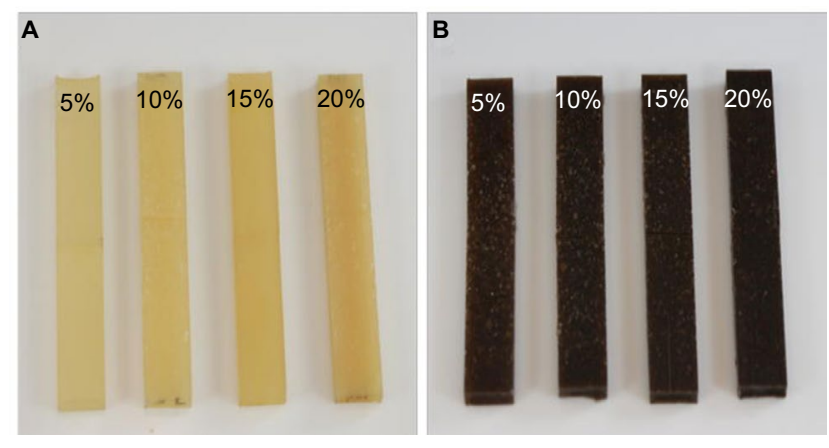

Figure 2 Uncoated and barium-coated HNTs (5\%-20\%).

Notes: (A) HNTs-doped PMMA cement. (B) Barium-coated HNTs-doped PMMA cement at 5\%. Increased HNT addition slightly darkens the PMMA cement. In contrast, barium-coated HNTs in PMMA cement results in a more dramatic color change.

Abbreviations: HNT, Halloysite nanotube; PMMA, poly(methyl methacrylate).

fact, $20 \mathrm{wt} \%$ HNTs added to the cement only increases the Hounsfield units to 164 compared to 68 for plain PMMA. Barium-coated HNTs at $15 \mathrm{wt} \%$ are $1029 \mathrm{HU}$. The bariumcoated HNTs were 6.3 times the contrast level of the control HNTs. The barium-coated HNTs, at $15 \mathrm{wt} \%$, were 15.1 times the contrast level of plain PMMA. The $15 \mathrm{wt} \%$ barium-coated HNTs had $\sim 70 \%$ of the contrast level of the commercial grade PMMA cement. Each $5 \mathrm{wt} \%$ increase in barium-coated HNTs resulted in a $300 \mathrm{HU}$ increase in contrast. It should be noted that multiple layers of barium coatings could be applied to further enhance the contrast level, and this approach may achieve a commercial level of contrast.

\section{Compression testing}

Compression testing was done on $6 \mathrm{~mm}$ diameter PMMA cylinders $(n=5)$ and consisted of control (PMMA-only), BA-mixed PMMA, HNTs/PMMA-only and BA-coated HNTs/PMMA groups following ASTM F-451 testing standards. This was done with percent additives ranging from $5 \%$ to $15 \%$ HNTs as shown in Figure 3. PMMA cements that were devoid of any additives had peak compression strength of $57.07 \mathrm{MPa}$. The addition of BA and BA-coated HNTs to PMMA cement did not cause any significant change in the compression strength of the PMMA cements.

The addition of lower percent concentrations of HNTs decreased the strength significantly when compared to PMMA cement only. At 15\% w/w of HNTs/PMMA, the strength was similar to that of regular PMMA.

\section{Flexural testing}

PMMA cement (with no additives) had a peak flexural strength of $81.3 \mathrm{MPa}$, while addition of BA and HNTs did not cause any significant change in flexural strength of the PMMA cement (Figure 4, $\alpha=0.05$.). In contrast, there was a significant change in flexural strength with addition of HNTs coated with BA $(\alpha=0.05)$. The addition of $15 \% \mathrm{w} / \mathrm{w}$ of barium-coated HNTs reduced the flexural strength. There was no significant change in strength with $5 \%, 7.5 \%$ and $10 \%$ HNT addition to PMMA cement (Figure 4). 


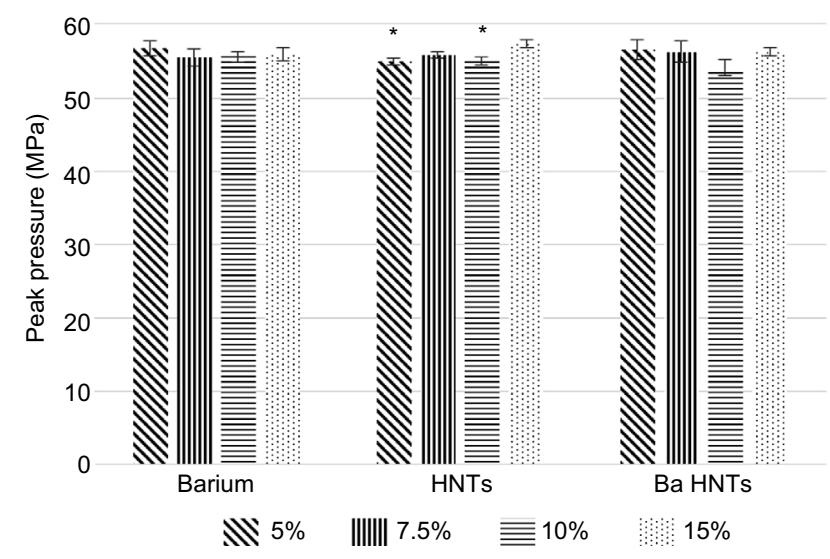

Figure 3 Compression testing of PMMA bone cement cylinders containing barium only, HNTs only or a composite of barium and HNTs (5\%-15\%).

Notes: Standard errors were calculated for each group and are shown as error bars. $n=5$. *Represents groups with significant difference at $\alpha=0.05$.

Abbreviations: PMMA, poly(methyl methacrylate); HNTs, Halloysite nanotubes; Ba HNTs, barium acetylacetonate-coated halloysite nanotubes.

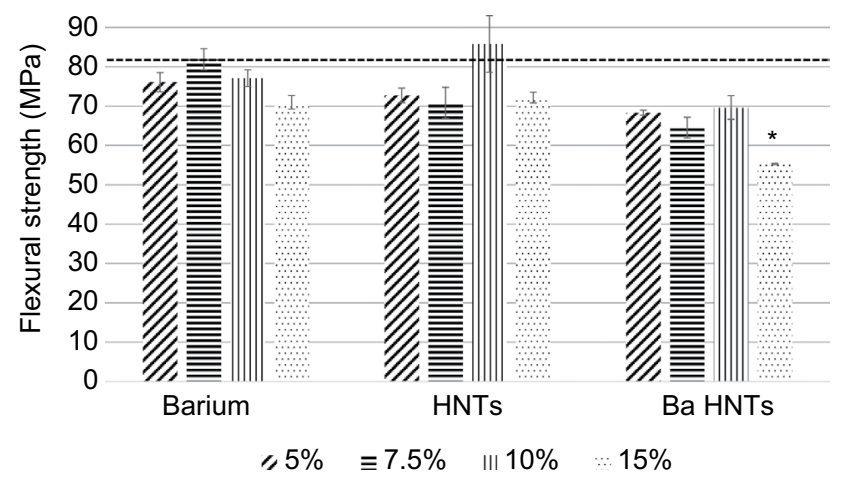

Figure 4 Flexural testing of PMMA bone cement cylinders containing barium only, HNTs only or a composite of barium and HNTs.

Notes: Dotted line in the graph represents the mean compression strength of control PMMA cement. Standard errors were calculated for each group and are shown as error bars, $n=5$. *Represents groups with significant difference at $\alpha=0.05$. Abbreviations: PMMA, poly(methyl methacrylate); HNTs, Halloysite nanotubes.

\section{Live/dead cytotoxicity assay}

Cells adhered and proliferated in all groups, but the cell density was more pronounced on scaffolds containing fillers. As seen in Figure 5, images taken after 24 hours in culture, live cells (green fluorescence) had broader cytoplasmic processes on scaffolds containing barium-coated HNTs, barium sulfate, HNTs and low-viscosity cements. The addition of these filler materials may be modifying the surface morphology of cements, favoring cell attachment and proliferation. A larger number of dead cells stained with red fluorescence were observed on the scaffolds containing HNTs compared with other groups. Increased cell density was observed on the scaffolds on day 4 as shown in Figure 6. Cells were observed to be large and denser on disks with barium-coated HNTs and low-viscosity cements. Cells cultured on nonbone cement disks were smaller, and the cell density was less compared with other groups.

\section{Cell proliferation}

Cell proliferation was estimated using XTT assay. Greater absorbance in the graph indicates larger number of viable cells. Disks were made with barium-coated HNTs, barium sulfate and HNTs at $10 \% \mathrm{w} / \mathrm{w}$. Osteosarcoma cells were incubated on the disks and analyzed after 24 hours (labeled as day 1 on the graph) and after 4 days in culture. There was no significant difference among PMMA cement samples with addition of barium sulfate, HNTs and barium-coated HNTs with respect to cell proliferation on both days of the assay (Figure 7). A significant difference in cell viability was observed when control cells were compared with disks doped with barium-loaded HNTs (Figure 7).

\section{Discussion}

Barium sulfate is commonly used as a radiopacifying agent. ${ }^{29}$ In this study, a method for making barium coatings on the surface of HNTs was created using BA.

Most metal acetylacetonates degrade completely at $300^{\circ} \mathrm{C}$, outgassing acetone and carbon dioxide and leaving behind positively charged metal ions. BA readily bonded to the negatively charged HNT outer surface allowing for a onestep sintered coating. SEM and EDX analyses indicated that the BA coating created a strong shell-like layer with spheroid depositions as revealed in scanning electron micrographs.

The addition of barium and HNTs did not cause any significant change in the flexural strength of PMMA cement. Moreover, there was no significant change in flexural strength with addition of BA-coated HNTs up to $10 \% \mathrm{w} / \mathrm{w}$ of PMMA. With $15 \% \mathrm{w} / \mathrm{w}$ BA-coated HNTs, a significant reduction in flexural strength was observed. Compression testing data also showed no significant difference between the controls, BA-PMMA and BA-coated HNT/PMMA. This suggests that our method can provide enhanced contrast without loss of the enhanced material strength. Wei et $\mathrm{al}^{24}$ also showed that gentamicin-doped HNTs added to PMMA could provide sustained gentamicin release without loss of material properties. ${ }^{17} \mathrm{In}$ fact, gains in tensile strength and adhesion were observed. Similar observations for barium additives have been reported in other studies. Khandaker et $\mathrm{al}^{42}$ reported on the modes of fracture for PMMA and PMMA-containing barium sulfate ceramic microparticles. Barium sulfate addition did not affect the strength of PMMA, although the addition of barium sulfate ceramic microparticles 

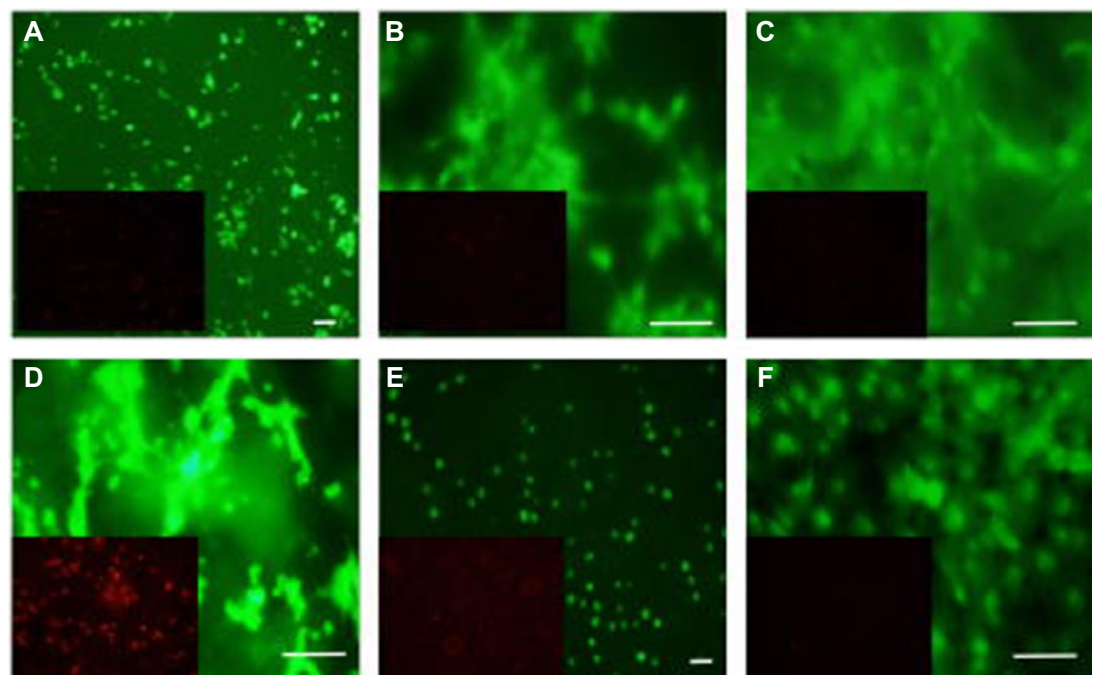

Figure 5 Live/dead cytotoxicity assay (day I in culture).

Notes: This assay was performed on control and experimental substrates after I day in culture. (A) Control cells; (B) Ba-coated HNTs I0\%; (C) barium I0\%; (D) HNTs 10\%; (E) PMMA and (F) low-viscosity cement. Scale bar=100 $\mu \mathrm{m}$. Insets show dead cells (red-orange fluorescence).

Abbreviations: HNTs, Halloysite nanotubes; PMMA, poly(methyl methacrylate).
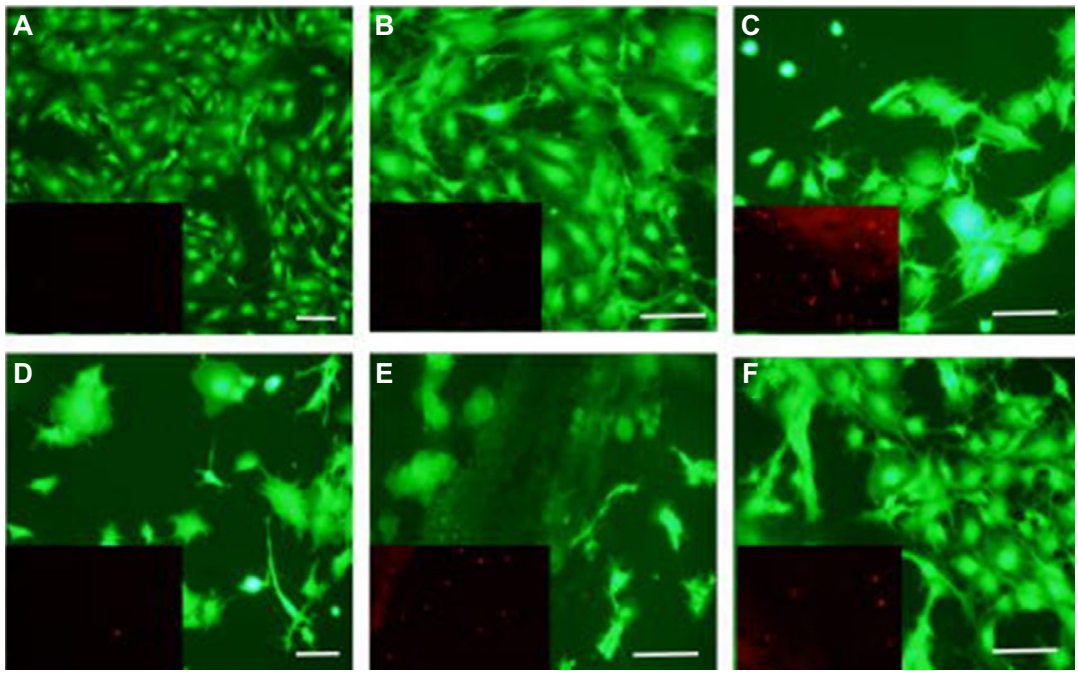

Figure 6 Live/dead cytotoxicity assay.

Notes: (A) Control cells; (B) Ba-coated HNTs 10\%; (C) barium 10\%; (D) HNTs 10\%; (E) PMMA and (F) low-viscosity cement. Scale bar=100 $\mu$ m. Insets show dead cells (red-orange fluorescence).

Abbreviations: HNTs, Halloysite nanotubes; PMMA, poly(methyl methacrylate).

made the PMMA more brittle. Similar observations have also been made by Gillani et $\mathrm{al},{ }^{43}$ although fracture strength and Young's modulus of PMMA and PMMA with barium sulfate were four to five times lower than those values reported in the study by Khandaker et al. ${ }^{42}$ The differences reported in material properties in these two studies may be due to the types of the mechanical tests used in these studies.

BA-coated HNTs were shown to be cytocompatible. The study by Khandaker et $\mathrm{al}^{42}$ also found no negative effect on cell viability after exposure to barium sulfate-doped PMMA. Barium sulfate-doped PMMA also produced less harmful exothermic reactions during solidification of PMMA and increased osteoblast adhesion. ${ }^{44}$ Wei et $\mathrm{al}^{24}$ also showed that gentamicin-doped HNTs reduced exothermic temperature during PMMA solidification after the addition of HNTs. Our long-term objective is to create a halloysite-based multifunctional platform that can provide a suite of properties, including sustained drug release, bioimaging capabilities 


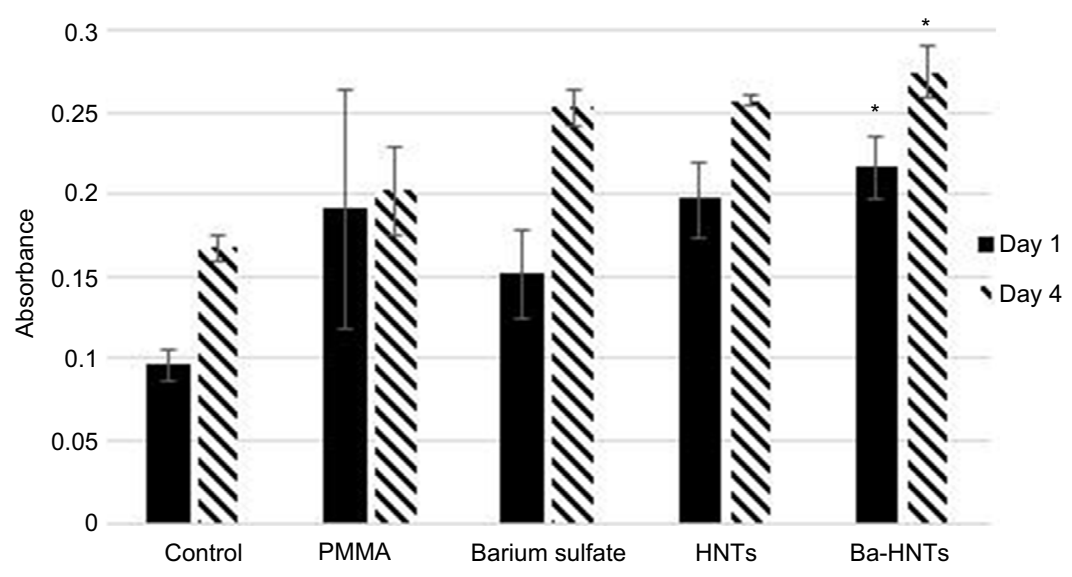

Figure 7 XTT analysis.

Notes: Osteosarcoma cells were incubated on the disks and analyzed after 24 hours and after 4 days in culture. $n=6$. *Represents groups with significant difference at $\alpha=0.05$. Abbreviations: PMMA, poly(methyl methacrylate); HNTs, Halloysite nanotubes; XTT, 2,3-bis-(2-methoxy-4-nitro-5-sulfophenyl)-2H-tetrazolium-5-carboxanilide.

and enhanced material properties. BA-coated HNTs, when added to PMMA bone cement, offer the potential for lower cost nanotechnology-based additives, and the sintering process creates, even more, customization opportunities. Preliminary studies with other metal acetate complexes such as iron, copper and gold have been conducted and show much promise and offer the potential to create highly customized HNT/PMMA materials. There is also the opportunity to provide bioactive additives through drug-doped BA-coated HNTs, thus overcoming the structural deficits as seen in the traditional methods of adding antibiotics or other additives to PMMA.

Another application area is the 3D printing of customized implants. Weisman ${ }^{45}$ demonstrated that the powdered component of commercial grade PMMA, including antibiotics and chemotherapeutics as additives, could be extruded at proper melt flow temperatures into a filament. ${ }^{31}$ The bioactive filaments were then $3 \mathrm{D}$ printed into beads and disks, and the additives retained their effective antimicrobial or cytostatic properties despite the heat required in this method of fabrication. The lack of the liquid monomer traditionally used to catalyze a standard reaction to make PMMA bone cement is not needed, thus reducing its potential toxicity. PMMA powder with additives, including but not limited to, barium and antibiotics, can be fabricated into a 3D printing filament and antimicrobial implants. However, they would still need to be surgically removed at some point since they do not degrade, necessitating additional surgeries. As many bioplastics and absorbable polymers are degradable, their advantage over PMMA would be not requiring a secondary surgery at a later date, thus reducing the impact on patient health. 3D fabrication can also offer an additional surface area for enhanced drug elution, smoother surfaces that will not damage tissue or break off compared with traditional hand-made antibiotic beads and the potential for combinatorial therapy.

\section{Conclusion}

Metal acetylacetonates were used to coat HNTs. SEM and EDX were used to verify whether HNTs were coated with BA. BA-coated HNTs improved radiopacity, strengthened native PMMA bone cement and were shown to be cytocompatible. The creation of barium-coated nanoparticles that provided improved contrast and material properties to PMMA offers the potential for enhanced imaging by radiologists or orthopedic surgeons. Further analysis of the BA-coated surface, however, is required to determine the evenness of BA-coating thickness, degree of surface adhesion and coating durability.

\section{Acknowledgment}

Funding for this project was supported by a Louisiana Governor's Biotechnology grant (awarded to David K Mills).

\section{Disclosure}

The authors report no conflicts of interest in this work.

\section{References}

1. DiMaio FR. The science of bone cement: a historical review. Orthopedics. 2002;25(12):1399.

2. Lewis G. Properties of acrylic bone cement: state of the art review. J Biomed Mater Res. 1997;38(2):155-182.

3. Magnan B, Bondi M, Maluta T, Samaila E, Schirru L, Dall'Oca C. Acrylic bone cement: current concept review. Musculoskelet Surg. 2013;97(2):93-100.

4. Charnley J. Anchorage of the femoral head prosthesis to the shaft of the femur. J Bone Joint Surg Br. 1960;42B:28-30. 
5. Breusch SJ, Kuhn KD. Bone cements based on polymethylmethacrylate. Orthopade. 2003;32:41-50.

6. Sugino A, Miyazaki T, Kawachi G, Kikuta K, Ohtsuki C. Relationship between apatite forming ability and mechanical properties of bioactive PMMA-based bone cement modified with calcium salts and alkoxysilane. J Mater Sci Mater Med. 2008;19(3):1399-1405.

7. Portigliatti-Barbos M, Rossi P, Salvadori L, Carando S, Gallinaro M. Bone-cement interface: a histological study of aseptic loosening in twelve prosthetic implants. Ital J Orthop Traumatol. 1986;12(4):499-505.

8. Bialoblocka-Juszczyk E, Baleani M, Cristofolini L, Viceconti M. Fracture properties of an acrylic bone cement. Acta Bioeng Biomech. 2008;10(1):21-26.

9. Saleh KJ, El Othmani MM, Tzeng TH, Mihalko WM, Chambers MC, Grupp TM. Acrylic bone cement in total joint arthroplasty: a review. J Orthop Res. 2016;34(5):737-744.

10. Grados F, Depriester C, Cayrolle G, Hardy N, Deramond H, Fardellone P. Long-term observations of vertebral osteoporotic fractures treated by percutaneous vertebroplasty. Rheumatology (Oxford). 2000;39(12):1410-1414.

11. Trout AT, Kallmes DF, Layton KF, Thielen KR, Hentz JG. Vertebral endplate fractures: an indicator of the abnormal forces generated in the spine after vertebroplasty. J Bone Miner Res. 2006;21(11):1797-1802.

12. Sharkey PF, Lichstein PM, Shen C, Tokarski AT, Parvizi J. Why are total knee arthroplasties failing today - has anything changed after 10 years? J Arthroplasty. 2014;29(9):1774-1778.

13. Lautenschlager EP, Marshall GW, Marks KE, Schwartz J, Nelson CL. Mechanical strength of acrylic bone cements impregnated with antibiotics. J Biomed Mater Res. 1976;10(6):837-845.

14. Frutos Cabanillas P, Diez Peña E, Barrales-Rienda JM, Frutos G. Validation and in vitro characterization of antibiotic-loaded bone cement release. Int J Pharm. 2000;209(1-2):15-26.

15. Lidgren L, Robertson O. Acrylic bone cements: clinical developments and current status: Scandinavia. Orthop Clin North Am. 2005;36(1):55-61.

16. Lissarrague MH, Fascio ML, Goyanes S, D'Accorso NB. Acrylic bone cements: the role of nanotechnology in improving osteointegration and tunable mechanical properties. J Biomed Nanotechnol 2014;10(12):3536-3557.

17. Lewis G. Properties of nanofiller-loaded poly (methyl methacrylate) bone cement composites for orthopedic applications: a review. J Biomed Mater Res B Appl Biomater. Epub 2016 Mar 10:doi: 10.1002/ jbm.b.33643.

18. Cisneros-Pineda OG, Cauich-Rodríguez JV, Cervantes-U JM, Vázquez B, San Román J. Combined influence of barium sulfate content and co-monomer concentration on properties of PMMA bone cements for vertebroplasty. J Biom Sci Poly. 2011;22(12):1563-1580.

19. Nien Y-H, Huang C-I. The mechanical study of acrylic bone cement reinforced with carbon nanotube. Mater Sci Eng B. 2010;B169: 134-137.

20. Ormsby R, McNally T, Mitchell C, Dunne N. Incorporation of multiwalled carbon nanotubes to acrylic based bone cements: effects on mechanical and thermal properties. J Mech Behav Biomed Mater. 2010;3(2):136-145.

21. Tana H, Aob H, Maa R, Tanga T. Quaternised chitosan-loaded polymethylmethacrylate bone cement: biomechanical and histological evaluations. J Orthop Trans. 2013;1(1):57-66.

22. Jiang H-J, Xu J, Qiu Z-Y, et al. Mechanical properties and cytocompatibility improvement of vertebroplasty PMMA bone cements by incorporating mineralized collagen. Materials. 2015;8:2616-2634.

23. Kwon SY, Cho EH, Kim SS. Preparation and characterization of bone cements incorporated with montmorillonite. J Biomed Mater Res $B$ Appl Biomater. 2007;83(1):276-284.
24. Wei W, Abdullayev E, Hollister A, Mills DK, Lvov Y. Clay nanotube/ poly(methyl methacrylate) bone cement composites with sustained antibiotic release. Macromol Mater Eng. 2012;297:645-653.

25. Zebarjad M, Saijadi A, Ebrahimi Sdrabadi T, et al. A study on mechanical properties of PMMA/hydroxyapatite nanocomposite. Engineering. 2011;3:795-801.

26. Albano C, Parra C, Gonzalez G. Comparison between different synthesis methods of PMMA/HA using ultrasonic radiation. Macromol Symp. 2010;290(1):95-106.

27. Khandaker M, Tarantini S. Material mismatch effect on the fracture of a bone-composite cement interface. Adv Mat Sci Appl. 2012;1(1):1-8.

28. Hang Chau JL, Hsieh C, Lin YM, Li AK. Preparation of transparent silica-PMMA nanocomposite hard coatings. Prog Poly Coatings. 2007;62(4):436-439.

29. Fu HP, Hong RY, Zhang YJ, et al. Preparation and properties investigation of PMMA/silica composites derived from silicic acid. Polym Adv Technol. 2009;20:84-91.

30. Kong, H, Jang J. Antibacterial properties of novel poly(methyl methacrylate) nanofiber containing silver nanoparticle. Langmuir. 2008;24(5):2051-2056.

31. Bordeepong S, Bhongsuwan D, Pungrassami T, Bhongsuwan T. Characterization of halloysite from thung yai district, Nakhon Si Thammarat Province, in Southern Thailand. Songklanakarin J Sci Technol. 2011;33(5):599-607.

32. Lvov YM, Shchukin DG, Möhwald H, Price RR. Halloysite clay nanotubes for controlled release of protective agents. ACS Nano. 2008;2(5):814-820.

33. Veerabadran NG, Price RR, Lvov YM. Clay nanotubes for encapsulation and sustained release of drugs. Nano. 2007;02:115-120.

34. Lvov Y, Abdullayev E. Functional polymer-clay nanotube composites with sustained release of chemical agents. Prog Polym Sci. 2013;38:1690-1719.

35. Sun L, Boyer C, Grimes RG, Mills DK. Drug coated clay nanoparticles for delivery of chemotherapeutics. Curr Nanosci. 2016;12:207-214.

36. Karnik S, Hines K, Mills DK. Nanoenhanced hydrogel system with sustained release capabilities.J Biomed Mater Res A. 2015;103(7):2416-2426.

37. Liu M, Guo B, Du M, Lei Y, Jia D. Natural inorganic nanotubes reinforced epoxy resin nanocomposites. J Polym Res. 2008;15:205-212.

38. Fernández A, Muniesa M, González J. Characterisation and processing of reinforced PA 6 with halloysite nanotubes (HNT) for injection molding. Strojniški Vest J Mech Eng. 2013;59(3):183-192.

39. Von Hoene J, Charles RG, Hickam WM. Thermal decomposition of metal acetylacetonates: mass spectrometer studies. J Phys Chem. 1958;62(9):1098-1101.

40. Pal B, Sharon M. Preparation of iron oxide thin film by metal organic deposition from $\mathrm{Fe}(\mathrm{III})$-acetylacetonate: a study of photocatalytic properties. Thin Solid Films. 2000;379:83-88.

41. Nicholson JC, Weisman JA, Boyer CJ, Wilson CG, Mills DK. Dry sintered metal coating of halloysite nanotubes. Appl Sci. 2016;6:265-270.

42. Khandaker M, Vaughn MB, Morris T, White J, Meng Z. Effect of additive particles on mechanical, thermal, and cell functioning properties of poly(methyl methacrylate) cement. Int $J$ Nanomedicine. 2014;9:2699-2712.

43. Gillani R, Ercan B, Qiao A, Webster TJ. Nanofunctionalized zirconia and barium-sulfate particles as bone cement additives. Intern J Nanomedicine. 2008;3(1):125-132.

44. Ricker A, Liu-Snyder P, Webster T. The influence of nano $\mathrm{MgO}$ and $\mathrm{BaSO} 4$ particle size addtives on properties of PMMA bone cement. Intern J Nanomedicine. 2010;5:1-11.

45. Weisman JA. Nanotechnology and Additive Manufacturing Platforms for Clinical Medicine: An Investigation of $3 D$ Printing Bioactive Constructs and Halloysite Nanotubes for Drug Delivery and Biomaterials. Louisiana Tech University; Ruston, LA: 2014. 
Nanotechnology, Science and Applications is an international, peer-reviewed, open access journal that focuses on the science of nanotechnology in a wide range of industrial and academic applications. It is characterized by the rapid reporting across all sectors, including engineering, optics, bio-medicine, cosmetics, textiles, resource sustainability and science. Applied research into nano-materials, particles, nano-structures and fabrication, diagnostics and analytics, drug delivery and toxicology constitute the primary direction of the journal. The manuscript management system is completely online and includes a very quick and fair peer-review system, which is all easy to use. Visit http://www.dovepress.com testimonials.php to read real quotes from published authors. 